ARTICLE

\title{
Superconductivity in an extreme strange metal
}

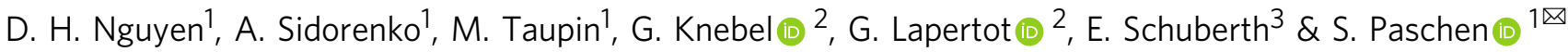

Some of the highest-transition-temperature superconductors across various materials classes exhibit linear-in-temperature 'strange metal' or 'Planckian' electrical resistivities in their normal state. It is thus believed by many that this behavior holds the key to unlock the secrets of high-temperature superconductivity. However, these materials typically display complex phase diagrams governed by various competing energy scales, making an unambiguous identification of the physics at play difficult. Here we use electrical resistivity measurements into the micro-Kelvin regime to discover superconductivity condensing out of an extreme strange metal state-with linear resistivity over 3.5 orders of magnitude in temperature. We propose that the Cooper pairing is mediated by the modes associated with a recently evidenced dynamical charge localization-delocalization transition, a mechanism that may well be pertinent also in other strange metal superconductors.

\footnotetext{
${ }^{1}$ Institute of Solid State Physics, Vienna University of Technology, Wiedner Hauptstr. 8-10, Vienna, Austria. ${ }^{2}$ Université Grenoble Alpes, CEA, Grenoble INP, IRIG, PHELIQS, Grenoble, France. ${ }^{3}$ Technische Universität München, Garching, Germany. ${ }^{凶}$ email: paschen@ifp.tuwien.ac.at
} 
$\mathrm{T}$ he phenomenon of superconductivity has fascinated scientists since its discovery in 1911. It had to await microscopic understanding, achieved in the BCS theory ${ }^{1}$, for almost 50 years. Conventional superconductors such as aluminum and niobium are now key players in the race for realizing the quantum computer ${ }^{2}$. Understanding superconductivity that goes beyond this framework, as first seen in the heavy fermion compound $\mathrm{CeCu}_{2} \mathrm{Si}_{2}{ }^{3}$ and since then considered for numerous materials classes ${ }^{4}$, is the next grand challenge.

In several of these "unconventional" superconductors-across high- $T_{\mathrm{c}}$ cuprates $^{5}$, iron pnictides and organic conductors ${ }^{6}$, heavy fermion metals ${ }^{7,8}$, and, very recently, infinite-layer nickelates ${ }^{9}$, twisted bilayer graphene ${ }^{10}$, and $\mathrm{WSe}_{2}{ }^{11}$-the normal state shows a linear-in-temperature "strange metal" electrical resistivity, at least in certain temperature ranges. This suggests that to decipher this type of superconductivity requires understanding the mechanism of the underlying strange metal normal state. However, because of the complexity of the phase diagrams of many of these systems, this has proven challenging. Multiple competing effects $^{4}$, crossovers between different scaling behaviors ${ }^{12}$, possible trivial linear-in-temperature resistivity contributions ${ }^{13}$, or simply the fact that strong superconductivity covers much of the normalstate phase space and needs to be suppressed by external parameters ${ }^{14}$, which may modify the original normal state, are inhibiting consensus on the key mechanism at play.

On the other hand, there is a material-the heavy fermion compound $\mathrm{YbRh}_{2} \mathrm{Si}_{2}{ }^{15}$-where such complications are absent and linear-in-temperature strange metal behavior ${ }^{15-18}$ has recently been pinned down as resulting from a dynamical electron localization-delocalization transition ${ }^{19}$ as the Kondo effect is destroyed at a magnetic quantum critical point $(\mathrm{QCP})^{20}$. Alas, this QCP appeared to lack superconductivity.

Our work unblocks this situation. By developing electrical resistivity measurements down to record low temperaturesmore than 1.5 orders of magnitude below state-of-the-art-and using them to study $\mathrm{YbRh}_{2} \mathrm{Si}_{2}$, we discover unconventional superconductivity condensing out of a further expanded range of strange metal behavior, now covering 3.5 orders of magnitude in temperature. This establishes the connection between electron localization-delocalization-derived strange metal behavior and superconductivity, discussed previously for several other materials $s^{21-24}$, to a new level of confidence, thereby pointing to its universality and putting the spotlight on Cooper pairing mediated by the critical modes that are associated with this transition.

We note that in purely itinerant systems at the border of an antiferromagnetic phase with spin density wave order, antiferromagnetic paramagnons ${ }^{25}$ may lead to deviations from Fermi liquid behavior (although generally not to strictly linear-intemperature resistivities) and provide superconducting pairing, a mechanism evoked for $\mathrm{CePd}_{2} \mathrm{Si}_{2}{ }^{26}$. However, for the above strange metals ${ }^{5-11}$ this mechanism seems unlikely, because no magnetic phase exists nearby and/or because there is evidence that this (weak-coupling) magnetic order parameter description is inappropriate.

The heavy fermion compound $\mathrm{YbRh}_{2} \mathrm{Si}_{2}$ exhibits a low-lying antiferromagnetic phase that is continuously suppressed to zero by a small magnetic field, establishing a field-induced QCP ${ }^{16}$. A linear temperature dependence of the electrical resistivity is seen below about $10 \mathrm{~K}^{16-18}$. This behavior is ruled out to be due to electron-phonon scattering because the non- $f$ reference material $\mathrm{LuRh}_{2} \mathrm{Si}_{2}$ is a normal metal and because Fermi liquid behavior is recovered when $\mathrm{YbRh}_{2} \mathrm{Si}_{2}$ is tuned away from the QCP by magnetic field ${ }^{16-18}$. The recent observation of quantum critical energy-over-temperature $(\omega / T)$ scaling in the charge dynamics ${ }^{19}$, together with jumps in the extrapolated zero-temperature Hall coefficient $^{27,28}$ and associated thermodynamic ${ }^{29}$ and spectroscopic signatures ${ }^{30}$, identifies a dynamical electron localization-delocalization transition as underlying the strange metal behavior.

\section{Results and discussion}

We have developed, and implemented in a nuclear demagnetization cryostat ${ }^{31}$, a setup for high-resolution electrical resistivity measurements to temperatures well below $1 \mathrm{mK}$ (see Methods), and used it to measure state-of-the-art $\mathrm{YbRh}_{2} \mathrm{Si}_{2}$ single crystals $^{17,32}$. To assess the previously discussed ${ }^{33}$ role of $\mathrm{Yb}$ nuclear moments, in addition to samples with natural abundance $\mathrm{Yb}$ (containing $14.2 \%{ }^{171} \mathrm{Yb}$ with a nuclear moment $I=1 / 2$ and $16.1 \%{ }^{173} \mathrm{Yb}$ with a nuclear moment $\left.I=5 / 2\right)^{32}$, we also studied ${ }^{174} \mathrm{YbRh}_{2} \mathrm{Si}_{2}$, which is free of nuclear $\mathrm{Yb}$ moments ${ }^{17}$. In zero magnetic field, both samples show the characteristic kink near the Néel temperature $T_{\mathrm{N}}$, as well as linear-in-temperature behavior above and Fermi liquid $T^{2}$ behavior below (Fig. 1a, b). The parameters extracted from these fits (Table 1) are in good agreement with previous results ${ }^{15-18}$, confirming the high reproducibility of the properties in state-of-the-art $\mathrm{YbRh}_{2} \mathrm{Si}_{2}$ single crystals. At the lowest temperatures, a pronounced drop of the resistivity signals the onset of superconductivity. It is fully displayed in Fig. 1c, d. In $\mathrm{YbRh}_{2} \mathrm{Si}_{2}$, the transition is rather sharp $\left(\Delta T_{\mathrm{c}} / T_{\mathrm{c}}=0.10\right.$ for a resistance change from $90 \%$ to $10 \%$ of the value above the transition), with an onset somewhat below $9 \mathrm{mK}$. In ${ }^{174} \mathrm{YbRh}_{2} \mathrm{Si}_{2}$, the onset is shifted to below $6 \mathrm{mK}$, and the transition is broadened, even though the residual resistance ratio of this sample is almost twice that of the normal $\mathrm{YbRh}_{2} \mathrm{Si}_{2}$ sample (Table 1), an effect that will be discussed later.

Application of magnetic fields within the $a$ - $a$ plane of the tetragonal crystal successively suppresses superconductivity in both samples (Fig. 1c, d). Note, however, that clear signs of superconductivity are visible even at the quantum critical field (see Fig. 1c, green curve, for $\mathrm{YbRh}_{2} \mathrm{Si}_{2}$, and Fig. $2 \mathrm{~b}$ for ${ }^{174} \mathrm{YbRh}_{2} \mathrm{Si}_{2}$ ), thus demonstrating superconductivity that nucleates directly out of the strange metal state (Fig. 2a, b). Our measurements expand the previously established strange metal regime to a record span of linear electrical resistivity over 3.5 orders of magnitude in temperature, with a high accuracy of 5\% in the linear exponent $\epsilon$ (Fig. 2c).

To characterize the superconductivity further, we performed isothermal magnetic field sweeps. In $\mathrm{YbRh}_{2} \mathrm{Si}_{2}$, all traces of superconductivity disappear only in fields beyond $70 \mathrm{mT}$, which is well above the quantum critical field of $60 \mathrm{mT}$ (Fig. 3a). The unusual two-step-like shape of the resistivity isotherms below 5 $\mathrm{mK}$, which is distinct from normal broadening in applied fields, suggests that two different superconducting phases might be involved. This is corroborated by the results on ${ }^{174} \mathrm{YbRh}_{2} \mathrm{Si}_{2}$, where the lowest-temperature isotherms show clear signs of reentrance (Fig. 3b). We note that no current dependence was observed, ruling out that flux-flow resistivity is at the origin of these characteristics. Next, we present color-coded temperature-magnetic field phase diagrams of $\mathrm{YbRh}_{2} \mathrm{Si}_{2}$ and ${ }^{174} \mathrm{YbRh}_{2} \mathrm{Si}_{2}$ (Fig. 3c, d), constructed from a large number of isotherms. Their merit is to give a general and fully unbiased impression of the phases present: an "S-shaped" superconducting region in $\mathrm{YbRh}_{2} \mathrm{Si}_{2}$ and two possibly separated superconducting regions in ${ }^{174} \mathrm{YbRh}_{2} \mathrm{Si}_{2}$.

For more quantitative phase diagrams, a definition of the (field-dependent) transition temperatures $T_{\mathrm{c}}$ and (temperaturedependent) upper critical fields $B_{\mathrm{c} 2}$ has to be adopted. We choose the midpoints of the resistive transitions, as sketched in Fig. 1c, d. For $\mathrm{YbRh}_{2} \mathrm{Si}_{2}$ this leads to the phase boundary delineated by the full $\left(B_{\mathrm{c} 2}\right)$ and open $\left(T_{\mathrm{c}}\right)$ blue circles in Fig. $4 \mathrm{a}$, which closely 
a
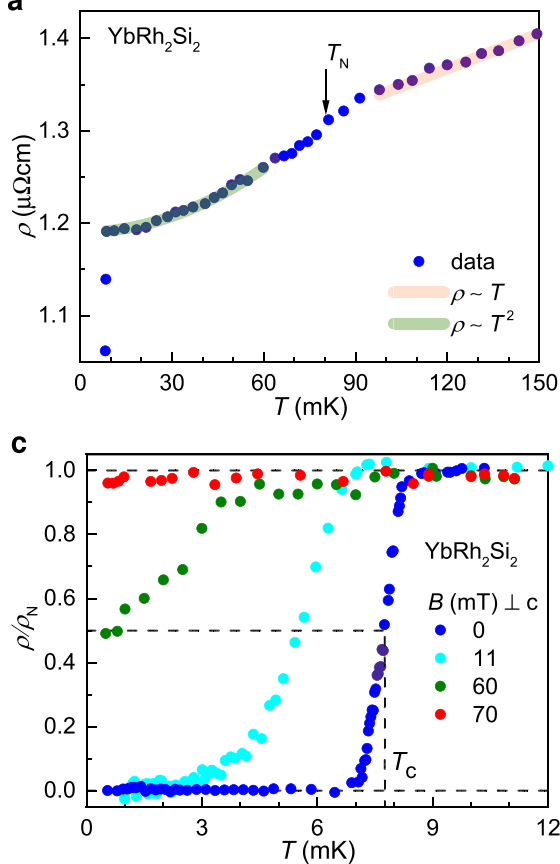

b

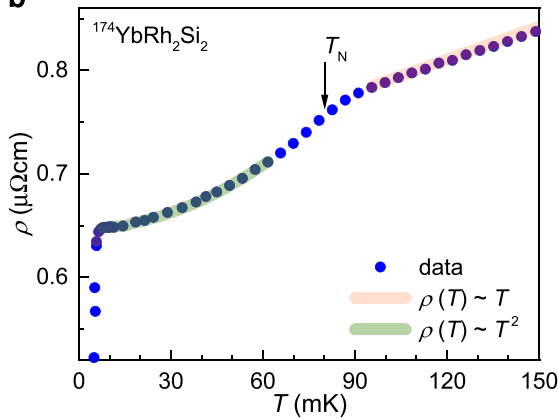

d

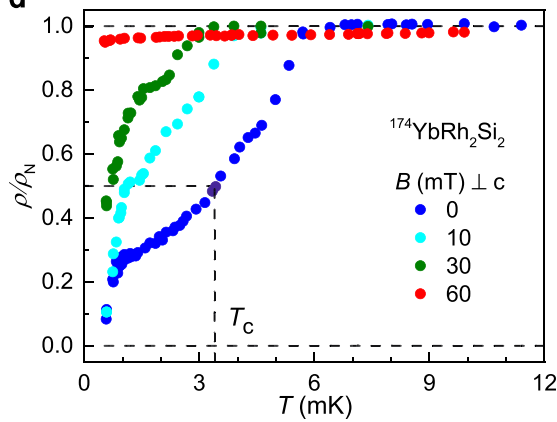

Fig. 1 Temperature dependence of the electrical resistivity of $\mathbf{Y} \mathbf{b} \mathbf{R h}_{\mathbf{2}} \mathbf{S i}_{\mathbf{2}}$ and ${ }^{174} \mathbf{Y} \mathbf{b} \mathbf{R} \mathbf{h}_{\mathbf{2}} \mathbf{S i}_{\mathbf{2}}$. $\mathbf{a}$, $\mathbf{b}$ Electrical resistivity $\rho(T)$ below $150 \mathrm{mK}$, showing linear-in$T$ behavior above the Neel temperature $T_{N}, T^{2}$ behavior below it, and the onset of superconductivity at the lowest temperatures. $\mathbf{c}$, $\mathbf{d}$ Electrical resistivity below $12 \mathrm{mK}$, scaled to its normal-state resistivity $\rho_{\mathrm{N}}$ just above the transition, showing the superconducting transition at $T_{\mathrm{c}}$, which we define as the temperature where $\rho(T)$ has dropped to $\rho_{\mathrm{N}} / 2$. Magnetic fields (applied within the tetragonal $a$ - $a$ plane) successively suppress $T_{\mathrm{c}}$. The 10 and $30 \mathrm{mT}$ curves for ${ }^{174} \mathrm{YbRh}_{2} \mathrm{Si}_{2}$ were extracted from isothermal field sweeps (Fig. 3b); all other curves were recorder as function of temperature.

Table 1 Characteristics of the investigated $\mathrm{YbRh}_{2} \mathrm{Si}_{2}$ and ${ }^{174} \mathrm{YbRh}_{2} \mathrm{Si}_{2}$ single crystals.

\begin{tabular}{llllllll} 
Sample & Batch & $\mathbf{R R R}_{\mathbf{1 0} \mathbf{~ m K}}$ & $\boldsymbol{\rho}_{\mathbf{0}}(\boldsymbol{\mu} \boldsymbol{\Omega} \mathbf{c m})$ & $\boldsymbol{A}\left(\boldsymbol{\mu} \mathbf{\Omega} \mathbf{c m} / \mathbf{K}^{2}\right)$ & $\rho_{\mathbf{0}}^{\prime}(\boldsymbol{\mu} \boldsymbol{\Omega} \mathbf{c m})$ & $\boldsymbol{A}^{\prime}(\boldsymbol{\mu} \mathbf{\Omega} \mathbf{c m} / \mathbf{K})$ & $\gamma_{\mathbf{K W}}^{\mathbf{T}}\left(\mathbf{J} / \mathbf{m o l} \mathbf{K}^{\mathbf{2}}\right)$ \\
\hline $\mathrm{YbRh}_{2} \mathrm{Si}_{2}$ & $63113 \_1$ & 67 & 1.19 & 20.2 & 1.23 & 1.17 & 1.42 \\
${ }_{174} \mathrm{YbRh}_{2} \mathrm{Si}_{2}$ & $\mathrm{Lap0288}$ & 123 & 0.55 & 14.8 & 0.59 & 0.85 & 1.22
\end{tabular}

Both samples are from batches studied in detail previously ${ }^{17,32}$. Their residual resistance ratios $\mathrm{RRR}_{10 \mathrm{mK}}=R(300 \mathrm{~K}) / R(10 \mathrm{mK})$, as well as the zero-field Fermi liquid behavior $\rho=\rho_{\mathrm{O}}+A T^{2}$ below $T_{\mathrm{N}}$ and the non-Fermi liquid behavior $\rho=\rho_{0}^{\prime}+A^{\prime} T$ at the quantum critical field of $60 \mathrm{mT}$ confirm high sample quality. To remove uncertainties in the geometric factors, we have assumed $\rho$ ( $\left.300 \mathrm{~K}\right)=80$ $\mu \Omega \mathrm{cm}^{15}$. The Sommerfeld coefficient in zero field $\gamma_{\mathrm{KW}}^{0 \mathrm{~T}}$, calculated from $A$ via the universal Kadowaki-Woods ratio $A / \gamma^{2}=10^{-5} \mu \Omega \mathrm{cm}\left(\mathrm{mol} \mathrm{K)^{2 }} /(\mathrm{mJ})^{2}\right.$, is a good estimate of the non-quantum critical contribution (see Supplementary Note 2: Estimates on Planckian dissipation).

resembles the shape drawn by the yellow color code $(50 \%$ resistance) in Fig. 3c. An initial rapid suppression of $T_{c}$ is followed by a much more gradual one, indicating that moving toward the QCP boosts the superconductivity against the general trend of field suppression associated with the Pauli- and/or orbitallimiting effect of the magnetic field (for cartoons of this field effect, see Supplementary Fig. 3). This is seen even more clearly in the $90 \%$ resistance line (boundary of the pale shading in Fig. $4 \mathrm{a}$ ) that exhibits a local maximum at a magnetic field only slightly below the QCP. This evidences that at least a component of the superconductivity of $\mathrm{YbRh}_{2} \mathrm{Si}_{2}$ is promoted by the same quantum critical fluctuations that are also responsible for the extreme strange metal behavior-thus anchoring both phenomena to the material's QCP. The fact that there might indeed be two distinct superconducting phases, one more readily suppressed by magnetic field and one that is less field sensitive, receives further support from the phase diagram of ${ }^{174} \mathrm{YbRh}_{2} \mathrm{Si}_{2}$, presented next.

Because the resistive transitions have finite widths, they interfere if two or more phase boundaries are nearby. For ${ }^{174} \mathrm{YbRh}_{2} \mathrm{Si}_{2}$, where the "unbiased" color-coded phase diagram already suggests two adjacent phases, we used a simple model to disentangle their effects (see Supplementary Note 1: Analysis of resistivity vs magnetic field isotherms and Supplementary Fig. 1). Indeed, by fitting this model to the data we find two distinct phases, a low-field one that we denote as phase I, and a fieldinduced one that we call phase II (Fig. $4 \mathrm{~b}$, see caption for the meaning of the different symbols). Again, we also show the $90 \%$ resistance line as the boundary of the pale shading. Using this criterion, phase I and II of ${ }^{174} \mathrm{YbRh}_{2} \mathrm{Si}_{2}$ grow together into a single superconducting region, similar to what is observed for $\mathrm{YbRh}_{2} \mathrm{Si}_{2}$. Conversely, this adds evidence to the above-proposed two-phase interpretation of the peculiarly shaped superconducting region of $\mathrm{YbRh}_{2} \mathrm{Si}_{2}$ (see cartoons in Supplementary Fig. 2). Despite the qualitative similarities between the phase diagrams of $\mathrm{YbRh}_{2} \mathrm{Si}_{2}$ and ${ }^{174} \mathrm{YbRh}_{2} \mathrm{Si}_{2}$, it is clear that quantitatively, the superconductivity is much weaker in ${ }^{174} \mathrm{YbRh}_{2} \mathrm{Si}_{2}$. Thus, whereas nuclear moments-present in $\mathrm{YbRh}_{2} \mathrm{Si}_{2}$ but absent in ${ }^{174} \mathrm{YbRh}_{2} \mathrm{Si}_{2}$ - are not a necessary ingredient to create superconductivity, they do considerably strengthen it.

In what follows we give a few simple estimates of characteristics of the superconductivity in $\mathrm{YbRh}_{2} \mathrm{Si}_{2}$ and ${ }^{174} \mathrm{YbRh}_{2} \mathrm{Si}_{2}$ (see Table 2). From the zero-field $T_{c}$ values (7.9 and $3.4 \mathrm{mK}$ for $\mathrm{YbRh}_{2} \mathrm{Si}_{2}$ and ${ }^{174} \mathrm{YbRh}_{2} \mathrm{Si}_{2}$, respectively) and upper critical field slopes $-d B_{\mathrm{c} 2} /\left.d T\right|_{T_{c}}(4.4$ and $2.1 \mathrm{~T} / \mathrm{K}$, much larger than in conventional superconductors), which we determined from linear fits in Fig. 4 (see red lines), we estimate the weak-coupling BCS Ginzburg-Landau coherence lengths $\xi_{\mathrm{GL}}$ (97 and $\left.215 \mathrm{~nm}\right)$. Together with the relevant (non-quantum critical) Sommerfeld 

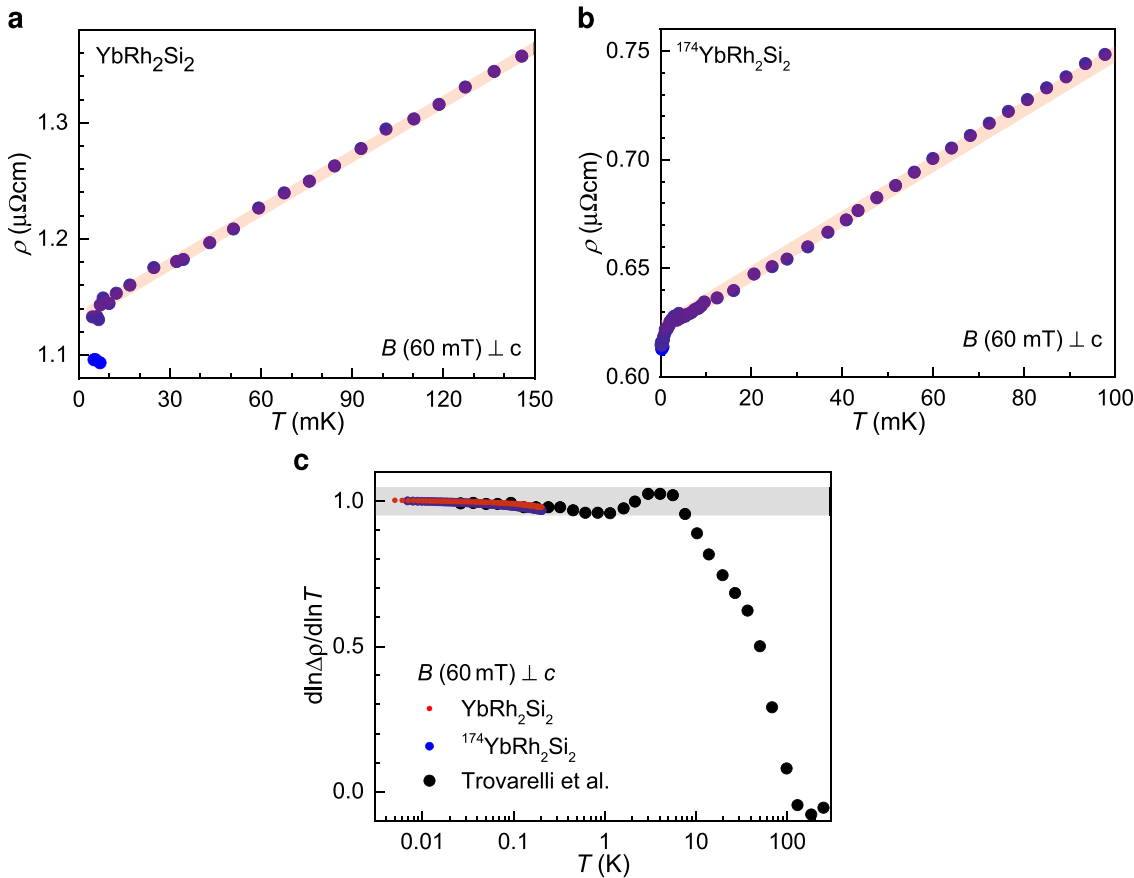

Fig. 2 Strange metal behavior of $\mathbf{Y b R h}_{\mathbf{2}} \mathbf{S i}_{\mathbf{2}}$ and ${ }^{{ }^{174}} \mathbf{Y} \mathbf{b} \mathbf{R h}_{\mathbf{2}} \mathbf{S i}_{\mathbf{2}}$. $\mathbf{a}, \mathbf{b}$ Temperature-dependent electrical resistivity at the quantum critical field of $60 \mathrm{mT}$ applied within the tetragonal $a-a$ plane. Superconductivity develops out of a strange metal $\rho \sim T$ normal state. The parameters of the linear-in- $T$ fits are given in Table 1. c Electrical resistivity exponent $\epsilon$ of the non-Fermi liquid form $\rho=\rho_{0}^{\prime}+A^{\prime} T^{\epsilon}$, determined as $\epsilon=\operatorname{dln} \Delta \rho / \operatorname{dln} T$, shown together with literature data up to high temperatures ${ }^{15}$. This establishes linear-in- $T$ resistivity, evidenced by $\epsilon=1 \pm 0.05$, over 3.5 orders of magnitude in temperature. Note that this presentation visualizes the temperature dependence of the exponent with great sensitivity, and that the error bar of \pm 0.05 corresponds to a high precision in the exponent's closeness to the value of 1 (for comparison see, e.g., Fig. 12 of ref. ${ }^{8}$ ).
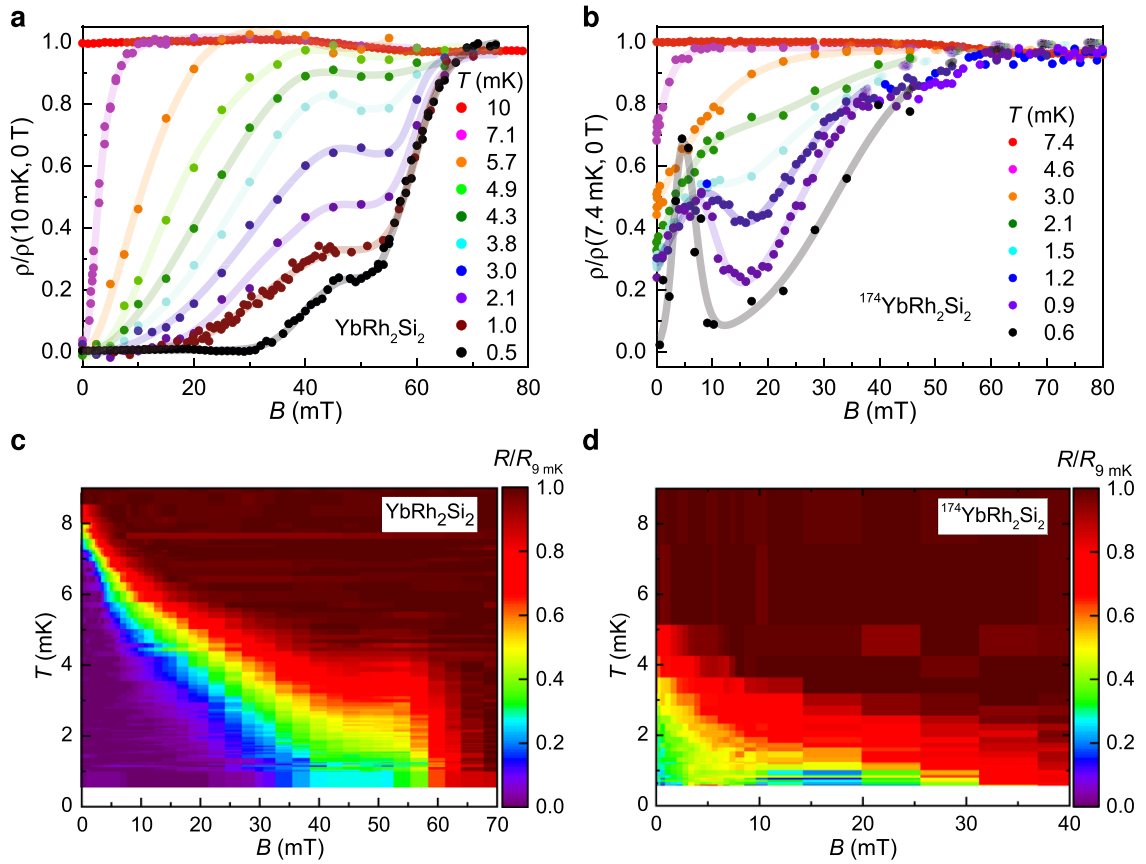

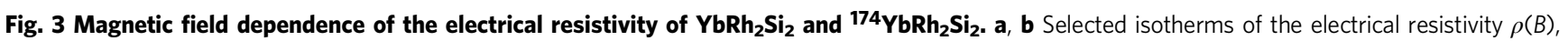
revealing reentrant superconductivity at low temperatures in ${ }^{174} \mathrm{YbRh}_{2} \mathrm{Si}_{2}(\mathbf{b})$ and remnants thereof in $\mathrm{YbRh}_{2} \mathrm{Si}_{2}$ (a). Lines are guides-to-the-eyes in (a) and fits to a multi-transition model (Supplementary Note 1: Analysis of resistivity vs magnetic field isotherms and Supplementary Fig. 1) in (b). c, d Temperature-magnetic field phase diagrams of the two samples, with the reduced electrical resistance $R / R(9 \mathrm{mK})$ as color code. The yellow color corresponds to the resistive midpoint. As a measure of $T_{c}(B)$ it indicates a phase boundary of unusual shape for $\mathrm{YbRh}_{2} \mathrm{Si}_{2}$, and two possibly distinct phases for ${ }^{174}{ }^{\mathrm{YbRh}} \mathrm{Si}_{2}$. 


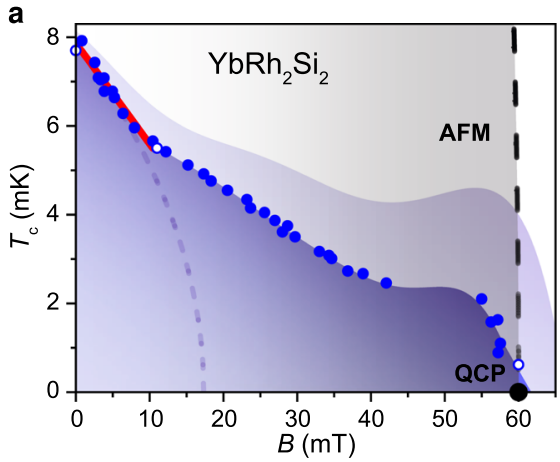

b

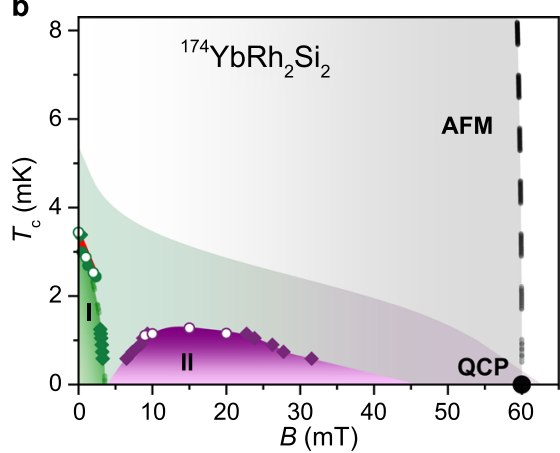

Fig. 4 Temperature-magnetic field phase diagrams of $\mathbf{Y b R h}_{\mathbf{2}} \mathbf{S i}_{\mathbf{2}}$ and ${ }^{174} \mathbf{Y b R h}_{\mathbf{2}} \mathbf{S i}_{\mathbf{2}}$. a Results for $\mathrm{YbRh}_{2} \mathrm{Si}_{2}$. $\mathbf{b}$ Results for ${ }^{174} \mathrm{YbRh} \mathrm{Si}_{2}$. Open ( $T_{\mathrm{c}}$ ) and full $\left(B_{\mathrm{c} 2}\right)$ circles of all colors represent midpoints (50\%) of the resistive transitions of iso- $B \rho(T)$ curves (Fig. 1c, d) T iso- $T \rho(B)$ curves (Fig. $2 a$, b), respectively. Full diamonds represent the $B_{\mathrm{c} 2}$ values extracted from a multi-transition model (Supplementary Note 1: Analysis of resistivity vs magnetic field isotherms and Supplementary Fig. 1) for the three involved transitions (green for leaving phase I, violet for entering and leaving phase II). The good agreement between diamonds and circles in the regions of minimal multi-phase interference justifies this procedure. The upper boundaries of the pale shaded areas away from data points represent $90 \%$ resistance lines. The black dashed line in both panels indicates the boundary of the antiferromagnetic (AFM) phase. It represents an extrapolation of data from refs. 28,29 , to $T=0$, the quantum critical point (QCP, black dot). The red lines are linear fits to the low-field (50\%) data, and determine both the upper critical field slopes $-d B_{c 2} /\left.d T\right|_{T_{c}}$ and the zero-field superconducting transition temperatures $T_{c}\left[=T_{c}(H=0)\right]$. The dashed lines represent (inverted) $B_{c 2}^{\prime}(T)=B_{c 2}^{\prime}(T=0) \cdot\left[1-\left(T / T_{c}\right)^{2}\right]$ mean-field curves determined from $-d B_{c 2} /\left.d T\right|_{T}$ and $T_{c}$, as estimates of the $($ lowfield) phase boundaries. Below $0.5 \mathrm{mK}$, all other phase/shading boundaries correspond to linear-in- $B$ extrapolations of fits to the lowest $T$ data points. Note that the perfect overlap of the full and open symbols demonstrates the high reproducibility of the results.

Table 2 Characteristics of the superconducting phase(s) in $\mathrm{YbRh}_{2} \mathrm{Si}_{2}$ and ${ }^{174} \mathrm{YbRh}_{2} \mathrm{Si}_{2}$.

\begin{tabular}{|c|c|c|c|c|c|c|c|c|c|}
\hline Sample & $T_{c}(m K)$ & $\left.\frac{-d B_{c 2}}{d T}\right|_{T_{c}}\left(\frac{T}{K}\right)$ & $\xi_{\mathrm{GL}}(\mathrm{nm})$ & $k_{F}\left(\frac{1}{n m}\right)$ & $I_{\mathrm{tr}}(\mathrm{nm})$ & $\lambda_{\mathrm{GL}}(\mu \mathrm{m})$ & $B_{\mathrm{c} 2}^{\prime}(\mathrm{mT})$ & $B_{p}(m T)$ & $\alpha$ \\
\hline $\mathrm{YbRh}_{2} \mathrm{Si}_{2}$ & 7.9 & 4.4 & 97 & 5.2 & 371 & 1.8 & 24 & 15 & 0.011 \\
\hline $174 \mathrm{YbRh}_{2} \mathrm{Si}_{2}$ & 3.4 & 2.1 & 215 & 4.8 & 976 & 2.0 & 5.0 & 6.4 & 0.0065 \\
\hline
\end{tabular}

Both $T_{c}(B)$ and $B_{c 2}(T)$ are defined as the midpoints of the resistive transitions (see Figs $1 c, d$ and $2 a, b$ ). The zero-field values $T_{c}\left[=T_{c}(H=0)\right]$ as well as the upper critical field slopes $-d B_{c 2} /\left.d T\right|_{T_{c}}$ are determined from linear fits to the data at small fields (red lines in Fig. 4). Listed are also estimates of the Ginzburg-Landau coherence length $\xi_{\mathrm{GL}}$, the average Fermi wavevector $k_{\mathrm{F}}$, the transport scattering length $I_{\text {tr, }}$ the Ginzburg-Landau penetration depth $\lambda_{G \text { L }}$ the (orbital limiting) upper critical field $B_{c 2}^{\prime}$, and the Pauli limiting field $B_{p}$ (see text). $\alpha$ is the prefactor of the scattering rate $1 / \tau=\alpha k_{B} T / \hbar$ of a simple Drude conductor, estimated from $T_{\mathrm{c}}, \xi_{\mathrm{GL}}$ (both below), and $\gamma_{\mathrm{KW}}^{\mathrm{O}}$ (from Table 1), as explained in the Supplementary Note 2: Estimates on Planckian dissipation; "Planckian dissipation" refers to $\alpha=1$.

coefficients (see Table 1) we derive the average Fermi wave vectors $k_{\mathrm{F}}\left(5.2\right.$ and $\left.4.8 \mathrm{~nm}^{-1}\right)$ and, with the residual resistivities $\rho_{0}$ (see Table 1), the transport scattering lengths $l_{\text {tr }}(371$ and $976 \mathrm{~nm}$ ) and the Ginzburg-Landau penetration depths $\lambda_{\mathrm{GL}}(1.8$ and 2.0 $\mu \mathrm{m})$. These describe moderately clean $\left(l_{\mathrm{tr}}>\xi_{\mathrm{GL}}\right)$, strongly type-II $\left(\lambda_{\mathrm{GL}} / \xi_{\mathrm{GL}} \gg 1 / \sqrt{2}\right)$ superconductivity. Estimates of the orbital and Pauli limiting upper critical fields, via $B_{\mathrm{c} 2}^{\prime}=$ $-0.7 T_{\mathrm{c}} d B_{\mathrm{c} 2} /\left.d T\right|_{T_{\mathrm{c}}}$ and $B_{\mathrm{p}}=1.764 k_{\mathrm{B}} T_{\mathrm{c}} /\left(\sqrt{2} \mu_{\mathrm{B}}\right)(24$ and $15 \mathrm{mT}$ for $\mathrm{YbRh}_{2} \mathrm{Si}_{2}$, and 5 and $6.4 \mathrm{mT}$ for ${ }^{174} \mathrm{YbRh}_{2} \mathrm{Si}_{2}$, respectively), might well be compatible with the phase boundary of phase I in ${ }^{174} \mathrm{YbRh}_{2} \mathrm{Si}_{2}$ and a putative corresponding low-field phase in $\mathrm{YbRh}_{2} \mathrm{Si}_{2}$. It is clear, however, that superconductivity in both $\mathrm{YbRh}_{2} \mathrm{Si}_{2}$ and ${ }^{174} \mathrm{YbRh}_{2} \mathrm{Si}_{2}$ extends to much larger fields (Fig. 4), providing further evidence for the unconventional nature of the observed superconductivity.

Next we discuss how our results relate to previous thermodynamic measurements on $\mathrm{YbRh}_{2} \mathrm{Si}_{2}$ with natural abundance $\mathrm{Yb}$, which provided evidence for superconductivity away from the QCP: shielding signals were detected, with onsets somewhat below $10 \mathrm{mK}$ and near $2 \mathrm{mK}$, in fields up to 0.055 and $0.418 \mathrm{mT}$, respectively ${ }^{33}$, leaving it open whether this superconductivity is related to the strange metal state of $\mathrm{YbRh}_{2} \mathrm{Si}_{2}$. The transition temperature we determine from zero-field electrical resistivity measurements on $\mathrm{YbRh}_{2} \mathrm{Si}_{2}$ (with an onset near $9 \mathrm{mK}$ ) is in good agreement with the upper transition (into the $\mathrm{B}$ phase) detected there, identifying it thus with our phase I. The observation of the lower transition (into the A + sc phase of ref. ${ }^{33}$ ) could then be taken as evidence that the two superconducting phases of $\mathrm{YbRh}_{2} \mathrm{Si}_{2}$ postulated above intersect (see sketch in Supplementary Fig. 2a). Alternatively, our low-field superconducting region could comprise both the B and sc phase of ref. ${ }^{33}$; then our high-field region would be a separate phase not detected in ref. ${ }^{33}$ (see sketch in Supplementary Fig. 2b), just as the high-field phase of ${ }^{174} \mathrm{YbRh}_{2} \mathrm{Si}_{2}$. This should be clarified by future magnetization/susceptibility measurements in lower fields (below the background field of $0.012 \mathrm{mT}$ reached in ref. ${ }^{33}$, which appears to be well above the lower critical field of the B phase), ideally on powdered samples to better assess the Meissner volume of the B phase.

The lower transition of ref. ${ }^{33}$ is accompanied by a specific heat anomaly that releases a giant amount of entropy. It was interpreted as phase transition into a state of hybrid nuclear and electronic spin order of $\mathrm{Yb}$, which reduces the internal (staggered) magnetic field of the primary electronic order and thus creates a less hostile environment for superconductivity. As our study reveals, the superconductivity in ${ }^{174} \mathrm{YbRh}_{2} \mathrm{Si}_{2}$, which lacks nuclear $\mathrm{Yb}$ moments and can thus not exhibit such hybrid order, is indeed considerably weaker than in $\mathrm{YbRh}_{2} \mathrm{Si}_{2}$. This confirms that nuclear $\mathrm{Yb}$ moments boost the superconductivity in $\mathrm{YbRh}_{2} \mathrm{Si}_{2}$. The fact that this mechanism apparently works up to temperatures well above the hybrid ordering temperature may be ascribed to short-range fluctuations, evidenced by the entropy release extending up to about 10 $\mathrm{mK}$. Apart from the overall weakening of the superconductivity in ${ }^{174} \mathrm{YbRh}_{2} \mathrm{Si}_{2}$ compared to $\mathrm{YbRh}_{2} \mathrm{Si}_{2}$, the phase diagrams of the two materials do, however, share many similarities (Fig. 4), calling for an understanding within the same framework.

The question that then arises is what is the mechanism of the Cooper pairing in the detected superconducting phases? 
We start by recapitulating our results that make the BCS mechanism extremely unlikely: (i) superconductivity in $\mathrm{YbRh}_{2} \mathrm{Si}_{2}$ condenses out of an extreme strange metal state, with linear-intemperature resistivity right down to the onset of superconductivity (Fig. 2a); (ii) the upper critical field slope (Table 2) as well as the directly measured critical field (Fig. 4a) strongly overshoot both the Pauli and the orbital limiting fields; (iii) the low-temperature resistivity isotherms exhibit a two-step transition (Fig. 3a), evidencing that one component is much less field sensitive than the other; (iv) the superconducting phase boundary deviates strongly from a mean-field shape (Figs $3 \mathrm{a}$ and $4 \mathrm{a}$ ), evidencing that the field boosts (at least part of) the superconductivity against the general trend of field suppression; (v) superconductivity is strongly suppressed by substituting the natural abundance $\mathrm{Yb}$ (of atomic mass 173.04 ) by ${ }^{174} \mathrm{Yb}$, though the isotope effect in a BCS picture would have a minimal effect (a reduction of $T_{c}$ by $\left.0.1 \%\right)$. It is thus natural to assume that quantum critical fluctuations are involved in stabilizing (at least the high-field part of) the superconductivity in $\mathrm{YbRh}_{2} \mathrm{Si}_{2}$.

Very recently, in a two-impurity Anderson model that features Kondo destruction ${ }^{20}$, the singlet-pairing susceptibility was found to be strongly enhanced by critical local moment fluctuations ${ }^{34}$. Because singlet pairing may be subject to Pauli limiting, this phase - though stabilized by quantum critical fluctuations - might be suppressed by the applied magnetic field even well before the QCP is reached. Thus, phase I of ${ }^{174} \mathrm{YbRh}_{2} \mathrm{Si}_{2}$ and the putative corresponding low-field phase of $\mathrm{YbRh}_{2} \mathrm{Si}_{2}$ are promising candidates for this type of superconductivity. Interestingly, in this context, unconventional superconductivity is also discussed in the spin-triplet channel ${ }^{35,36}$. It is tempting to consider phase II of ${ }^{174} \mathrm{YbRh}_{2} \mathrm{Si}_{2}$ and the putative corresponding high-field phase of $\mathrm{YbRh}_{2} \mathrm{Si}_{2}$ to be of this kind, which would provide a compelling link to recently discovered candidate spin-triplet topological superconductors ${ }^{37,38}$. Of course, any model for superconductivity in $\mathrm{YbRh}_{2} \mathrm{Si}_{2}$ (and ${ }^{174} \mathrm{YbRh}_{2} \mathrm{Si}_{2}$ ) should also correctly capture the normal state properties.

At the QCP of $\mathrm{YbRh}_{2} \mathrm{Si}_{2}$, a dynamical electron localization-delocalization transition, featuring both linear-intemperature dc resistivity, and linear-in-frequency and linear-intemperature "optical resistivity", was evidenced by quantum critical frequency-over-temperature scaling, with a critical exponent of 1 , of the $\mathrm{THz}$ conductivity of $\mathrm{YbRh}_{2} \mathrm{Si}_{2}{ }^{19}$. This evidences critical modes in addition to the ones associated with the suppression of the (antiferro)magnetic order parameter. A microscopic mechanism compatible with this scaling is the disentanglement of the (electronic) $\mathrm{Yb}$ if moments from the conduction electrons as static Kondo screening breaks down at the QCP ${ }^{19,20,27-30}$. Whether spintriplet superconductivity may arise in models that capture this physics remains to be clarified by future work. Given that the quantum critical magnetic field is considerably larger than the scale associated with the superconducting transition temperature near the QCP, a promising direction is to consider the role of the applied magnetic field as reducing the spin symmetry from being in-plane continuous to Ising-anisotropic. In fact, calculations in an Isinganisotropic two-impurity Bose-Fermi Anderson model suggest that near the model's Kondo-destruction QCP the spin-triplet pairing correlation is competitive with the spin-singlet one ${ }^{39}$. Naturally, the proposal of spin-triplet superconductivity should also be scrutinized by future experiments, including probes of anisotropies and NMR investigations, which are in principle feasible at ultralow temperatures.

Our observation of unconventional superconductivity condensing out of the textbook strange metal state of $\mathrm{YbRh}_{2} \mathrm{Si}_{2}$ ends a debate about reasons for its (previously supposed) absence. Thus, neither the $4 f$ moment stemming from $\mathrm{Yb}$ or the tuning parameter being magnetic field (as opposed to the typical situation of Ce-based systems under pressure or doping tuning), nor the QCP being governed by effects beyond order parameter fluctuations inhibits superconductivity. Instead, we propose that critical modes associated with a dynamical electron localization-delocalization transition mediate the strange-metal unconventional superconductivity in $\mathrm{YbRh}_{2} \mathrm{Si}_{2}$. Future experiments, ideally in conjunction with ab initio-based theoretical work, shall ascertain this assignment, disentangle the different superconducting phases, determine the symmetry of the order parameter, clarify further important details such as the single vs multiband nature of the superconductivity, and even explore the possibility of exotic surface phases.

Finally, we relate our discovery to strange-metal unconventional superconductors in other materials classes. It has been pointed out that the strange metal behavior in many of these is compatible with "Planckian dissipation", i.e., with the transport scattering rate $1 / \tau$ in a simple Drude conductor being equal to $\alpha k_{\mathrm{B}} T / \hbar$, with $\alpha \approx 1^{40}$. For $\mathrm{YbRh}_{2} \mathrm{Si}_{2}$ and ${ }^{174} \mathrm{YbRh}_{2} \mathrm{Si}_{2}$, we estimate much smaller $\alpha$ values (see Table 2, and Supplementary Note 2: Estimates on Planckian dissipation), suggesting that linear-in-temperature resistivity-even in the extreme form observed here-does not require the Planckian limit to be reached. Whether this is related to the very strongly correlated nature of this compound (with effective masses above $1000 \cdot m_{0}$, much beyond those in the materials considered in ref. ${ }^{40}$ ) or its pronounced deviation from Drude-like behavior (see Supplementary Materials of ref. ${ }^{19}$ ) is an interesting question for future studies. As to a microscopic understanding of the phenomenon, we point out that charge delocalization transitions, similar to those observed in $\mathrm{YbRh}_{2} \mathrm{Si}_{2}$, are being discussed also in other strangemetal superconductors, including the high- $T_{c}$ cuprates $^{21,22}$, an organic conductor ${ }^{23}$ and, tentatively, even magic angle bilayer graphene ${ }^{24}$. Our results thus point to the exciting possibility that a dynamical electron localization-delocalization transition may mediate strange-metal unconventional superconductivity in a broad range of materials classes.

\section{Methods}

Refrigerator and thermometry. All measurements were carried out in the Vienna nuclear demagnetization refrigerator ${ }^{31}$. We use resistance thermometry (Pt resistance thermometers Pt-1000, $\mathrm{RuO}_{2}$ thermometers, and sliced Speer carbon resistance thermometers) for temperatures above $10 \mathrm{mK}$ and magnetic thermometry (CMN, pulsed Pt-NMR thermometers, via the pulsed nuclear magnetic resonance of ${ }^{195} \mathrm{Pt}$ nuclei) for temperature below $20 \mathrm{mK}$. For the presented results, we used a CMN thermometer between 20 and $2 \mathrm{mK}$, a Speer thermometer above, and a PtNMR thermometer below.

Filters. To attenuate radiofrequency radiation, a series of filters and thermalization stages consisting of thermo-coax cables (from room temperature to the $50 \mathrm{mK}$ plate), silver-epoxy filters, and RC filters were installed. The silver-epoxy filters are also used for thermalization (down to the mixing chamber temperature).

Sample holders and thermalization. Machined silver sample holders made from a $5 \mathrm{~N}$ silver rod were annealed to reach a residual resistance ratio of 2000 . They are directly screwed to the nuclear stage using home-made silver screws. To thermalize the samples, one of the two outer contact points (of the 4-point technique, see point E below) was connected by $50 \mu \mathrm{m}$ gold wires to the silver holder, via spot welding on the sample and screwing to the silver holder. A separate grounding point of excellent quality (anchored $600 \mathrm{~m}$ below ground level) was used. The different stages of the cryostat ( 4 and $1 \mathrm{~K}$ plate, still, $50 \mathrm{mK}$ plate, mixing chamber, copper nuclear stage) were all connected via $\mathrm{NbTi} / \mathrm{CuNi}$ superconducting wires to the same ground, such that the ground potential was highly uniform. To avoid ground loops, all measurements devices were connected via opto-couplers. During the measurements at ultralow temperatures (below $10 \mathrm{mK}$ ), all measurement devices were powered by $4 \times 12 \mathrm{~V}-150 \mathrm{~A}$ batteries with floating ground. For electrical isolation (away from the ground point), Vespel was used on the sample holder, the superconducting coil, and the Nb-superconducting shield.

Magnetic field applied to the samples. A dc magnetic field coil made of superconducting $\mathrm{NbTi}$ wire $\left(T_{\mathrm{c}}=9.2 \mathrm{~K}\right)$, a superconducting $\mathrm{Nb}$ cylinder $(20 \mathrm{~mm}$ diameter, $10 \mathrm{~cm}$ length), and the sample holder are concentrically assembled. The field coil and the superconducting shield are thermalized to the mixing chamber, 
the sample holder to the nuclear stage. Magnetic fields at the position of the samples up to $80 \mathrm{mT}$ were generated by applying a dc current, with the precision of a few $\mu \mathrm{T}$. For the highest fields, a current above 1.4 A was passed through the selfmade coil. Because this represented a considerable risk of quenching and breaking the magnet, only a final set of experiments was done up to the highest fields. In particular, most of the measurements on ${ }^{174} \mathrm{YbRh}_{2} \mathrm{Si}_{2}$ were done up to $45 \mathrm{mT}$ only.

Resistivity measurements. Electrical resistivity measurements were done with a 7124 Precision Lock-in Amplifier. Electrical currents were sourced by a CS580 voltage-controlled current source. The lowest measurement current in our experiments was $10 \mathrm{nA}$. To increase the signal to noise ratio, low-temperature transformers (encapsulated in a lead shield) with a gain of 1000 were used. They were installed at and thermalized to the mixing chamber. In addition, a SR560 low noise voltage pre-amplifier was used at room temperature. Electrical contacts for these measurements were made by spot-welding gold wires to the samples, in a standard 4-point geometry. Carefully derived measurement protocols (electrical current densities, sweep rates, waiting times, etc.) for a good thermalization of the samples to the nuclear stage were followed for all presented measurements; the good thermalization is confirmed by the reproducibility of the results between different types of experiments (e.g., temperature vs magnetic-field sweeps, cooling vs warming curves). Applying larger currents leads to overheating effects at the lowest temperatures, but no evidence for flux-flow resistance could be revealed.

\section{Data availability}

The datasets generated during and/or analyzed during the current study are available from the corresponding author on reasonable request.

Received: 7 December 2020; Accepted: 28 June 2021;

Published online: 21 July 2021

\section{References}

1. Bardeen, J., Cooper, L. N. \& Schrieffer, J. R. Theory of superconductivity. Phys. Rev. 108, 1175 (1957).

2. Wendin, G. Quantum information processing with superconducting circuits: a review. Rep. Prog. Phys. 80, 106001 (2017).

3. Steglich, F. et al. Superconductivity in the presence of strong Pauli paramagnetism: $\mathrm{CeCu}_{2} \mathrm{Si}_{2}$. Phys. Rev. Lett. 43, 1892 (1979).

4. Stewart, G. R. Unconventional superconductivity. Adv. Phys. 66, 75 (2017)

5. Legros, A. et al. Universal $T$-linear resistivity and Planckian dissipation in overdoped cuprates. Nat. Phys. 15, 142 (2019).

6. Doiron-Leyraud, $N$. et al. Correlation between linear resistivity and $T_{c}$ in the Bechgaard salts and the pnictide superconductor $\mathrm{Ba}\left(\mathrm{Fe}_{1-\mathrm{x}} \mathrm{Co}_{\mathrm{x}}\right)_{2} \mathrm{As}_{2}$. Phys. Rev. B 80, 214531 (2009).

7. Park, T. et al. Isotropic quantum scattering and unconventional superconductivity. Nature 456, 366 (2008).

8. Knebel, G., Aoki, D., Brison, J.-P. \& Flouquet, J. The quantum critical point in CeRhIn $_{5}$ : a resistivity study. J. Phys. Soc. Jpn. 77, 114704 (2008).

9. Li, D. et al. Superconductivity in an infinite-layer nickelate. Nature 572, 624 (2019).

10. Cao, Y. et al. Strange metal in magic-angle graphene with near Planckian dissipation. Phys. Rev. Lett. 124, 076801 (2020).

11. Wang, L. et al. Correlated electronic phases in twisted bilayer transition metal dichalcogenides. Nat. Mater. 19, 861 (2020).

12. van der Marel, D. et al. Quantum critical behaviour in a high $T_{c}$ superconductor. Nature 425, 271 (2003).

13. Polshyn, H. et al. Large linear-in-temperature resistivity in twisted bilayer graphene. Nat. Phys. 15, 1011 (2019).

14. Proust, C. \& Taillefer, L. The remarkable underlying ground states of cuprate superconductors. Annu. Rev. Condens. Matter Phys. 10, 409-429 (2019).

15. Trovarelli, $\mathrm{O}$. et al. $\mathrm{YbRh}_{2} \mathrm{Si}_{2}$ : pronounced non-Fermi-liquid effects above a low-lying magnetic phase transition. Phys. Rev. Lett. 85, 626 (2000).

16. Gegenwart, P. et al. Magnetic-field induced quantum critical point in $\mathrm{YbRh}_{2} \mathrm{Si}_{2}$. Phys. Rev. Lett. 89, 056402 (2002).

17. Knebel, G. et al. Localization of $4 f$ state in $\mathrm{YbRh}_{2} \mathrm{Si}_{2}$ under magnetic field and high pressure: comparison with $\mathrm{CeRh}_{2} \mathrm{Si}_{2}$. J. Phys. Soc. Jpn. 75, 114709 (2006).

18. Taupin, M. et al. Thermal conductivity through the quantum critical point in $\mathrm{YbRh}_{2} \mathrm{Si}_{2}$ at very low temperature. Phys. Rev. Lett. 115, 046402 (2015).

19. Prochaska, L. et al. Singular charge fluctuations at a magnetic quantum critical point. Science 367, 285 (2020).

20. Si, Q., Rabello, S., Ingersent, K. \& Smith, J. Locally critical quantum phase transitions in strongly correlated metals. Nature 413, 804 (2001)

21. Balakirev, F. F. et al. Signature of optimal doping in Hall-effect measurements on a high-temperature superconductor. Nature 424, 912 (2003).

22. Badoux, S. et al. Change of carrier density at the pseudogap critical point of a cuprate superconductor. Nature 531, 210-214 (2016).
23. Oike, H., Miyagawa, K., Taniguchi, H. \& Kanoda, K. Pressure-induced Mott transition in an organic superconductor with a finite doping level. Phys. Rev. Lett. 114, 067002 (2015).

24. Cao, Y. et al. Unconventional superconductivity in magic-angle graphene superlattices. Nature 556, 43 (2018)

25. Scalapino, D. J. A common thread: the pairing interaction for unconventional superconductors. Rev. Mod. Phys. 84, 1383 (2012).

26. Mathur, N. et al. Magnetically mediated superconductivity in heavy fermion compounds. Nature 394, 39 (1998).

27. Paschen, S. et al. Hall-effect evolution across a heavy-fermion quantum critical point. Nature 432, 881 (2004).

28. Friedemann, S. et al. Fermi-surface collapse and dynamical scaling near a quantum-critical point. Proc. Natl Acad. Sci. U. S. A. 107, 14547 (2010).

29. Gegenwart, P. et al. Multiple energy scales at a quantum critical point. Science 315, 969 (2007).

30. Seiro, S. et al. Evolution of the Kondo lattice and non-Fermi liquid excitations in a heavy-fermion metal. Nat. Commun. 9, 3324 (2018).

31. Nguyen, D. H. et al. The Vienna nuclear demagnetization refrigerator. J. Phys. Conf. Ser. 400, 052024 (2012).

32. Krellner, C., Taube, S., Westerkamp, T., Hossain, Z. \& Geibel, C. Single-crystal growth of $\mathrm{YbRh}_{2} \mathrm{Si}_{2}$ and $\mathrm{YbIr}_{2} \mathrm{Si}_{2}$. Philos. Mag. 92, 2508 (2012).

33. Schuberth, E. et al. Emergence of superconductivity in the canonical heavyelectron metal $\mathrm{YbRh}_{2} \mathrm{Si}_{2}$. Science 351, 485 (2016).

34. Cai, A., Pixley, J. H., Ingersent, K. \& Si, Q. Critical local moment fluctuations and enhanced pairing correlations in a cluster Anderson model. Phys. Rev. B 101, 014452 (2020)

35. Kang, J., Fernandes, R. M., Abrahams, E. \& Wölfle, P. Superconductivity at an antiferromagnetic quantum critical point: Role of energy fluctuations. Phys. Rev. B 98, 214515 (2018).

36. Li, Y., Wang, Q., Xu, Y., Xie, W. \& Yang, Y.-f. Nearly degenerate $p_{x}+i p_{y}$ and $d_{x^{2}-y^{2}}$ pairing symmetry in the heavy fermion superconductor $\mathrm{YbRh}_{2} \mathrm{Si}_{2}$. Phys Rev. B 100, 085132 (2019).

37. Zhang, P. et al. Observation of topological superconductivity on the surface of an iron-based superconductor. Science 360, 182 (2018).

38. Ran, S. et al. Nearly ferromagnetic spin-triplet superconductivity. Science 365, 684 (2019).

39. Pixley, J. H., Deng, L., Ingersent, K. \& Si, Q. Pairing correlations near a Kondo-destruction quantum critical point. Phys. Rev. B 91, 201109 (2015).

40. Bruin, J. A. N., Sakai, H., Perry, R. S. \& Mackenzie, A. P. Similarity of scattering rates in metals showing $T$-linear resistivity. Science 339, 804 (2013).

\section{Acknowledgements}

The authors thank A. Casey, Ch. Enss, G. Frossatti, L. Levitin, J. Saunders, A. de Waard, D. Zumbuehl, and other colleagues from the European Microkelvin Platform (EMP) for sharing expertise in ultralow-temperature techniques, C. Krellner for growing $\mathrm{YbRh}_{2} \mathrm{Si}_{2}$ single crystals under supervision of Ch. Geibel and F. Steglich in Dresden, M. Brando for assistance in the selection of suitable single crystals, and P. Buehler, A. Prokofiev, Q. Si, and F. Steglich for fruitful discussions. The team in Vienna acknowledges financial support from the Austrian Science Fund (FWF grants P29296-N27 and DK W1243), the EMP (H2020 Project 824109), and the European Research Council (ERC Advanced Grant 227378).

\section{Author contributions}

S.P. initiated and led the study. G.K. and G.L. synthesized and characterized the ${ }^{174} \mathrm{YbRh}_{2} \mathrm{Si}_{2}$ single crystals. D.H.N. and A.S. set up the ultralow-temperature experiments, with initial guidance by E.S., and performed the measurements. D.H.N., M.T., and S.P. analyzed the data. S.P. wrote the manuscript, with input from all authors. All authors contributed to the discussion.

\section{Competing interests}

The authors declare no competing interests.

\section{Additional information}

Supplementary information The online version contains supplementary material available at https://doi.org/10.1038/s41467-021-24670-z.

Correspondence and requests for materials should be addressed to S.P.

Peer review information Nature Communications thanks the anonymous reviewers for their contribution to the peer review of this work. Peer reviewer reports are available.

Reprints and permission information is available at http://www.nature.com/reprints

Publisher's note Springer Nature remains neutral with regard to jurisdictional claims in published maps and institutional affiliations. 
(c) (i) Open Access This article is licensed under a Creative Commons Attribution 4.0 International License, which permits use, sharing, adaptation, distribution and reproduction in any medium or format, as long as you give appropriate credit to the original author(s) and the source, provide a link to the Creative Commons license, and indicate if changes were made. The images or other third party material in this article are included in the article's Creative Commons license, unless indicated otherwise in a credit line to the material. If material is not included in the article's Creative Commons license and your intended use is not permitted by statutory regulation or exceeds the permitted use, you will need to obtain permission directly from the copyright holder. To view a copy of this license, visit http://creativecommons.org/ licenses/by/4.0/.

(C) The Author(s) 2021 